\title{
A REMARK ON THE EXISTENCE OF POSITIVE PERIODIC SOLUTIONS OF SUPERLINEAR PARABOLIC PROBLEMS
}

\author{
MARIA J. ESTEBAN
}

(Communicated by Walter Littman)

ABSTRACT. We prove the existence of a solution for the following problem:

$$
\begin{aligned}
& \partial_{t} u-\Delta u=f(t, x, u) \quad \text { in }(0, T) \times \Omega, \quad u>0 \quad \text { in }(0, T) \times \Omega, \\
& u(T)=u(0) \quad \text { in } \Omega, \quad u=0 \quad \text { on }(0, T) \times \partial \Omega,
\end{aligned}
$$

where $\Omega$ is a bounded domain of $R^{N}$ and the function $f(t, x, \cdot)$ grows more slowly than $u^{\alpha}$ at $+\infty$, with $\alpha<N /(N-2)$.

RESUMÉ. On démontre ici l'existence d'une solution positive pour le problème parabolique périodique suivant

$$
\begin{aligned}
& \partial_{t} u-\Delta u=f(t, x, u) \quad \text { in }(0, T) \times \Omega, \quad u>0 \quad \text { in }(0, T) \times \Omega, \\
& u(T)=u(0) \quad \text { in } \Omega, \quad u=0 \quad \text { on }(0, T) \times \partial \Omega,
\end{aligned}
$$

où $\Omega$ est un domaine borné de $R^{N}$ et la fonction $f(t, x, \cdot)$ croit plus lentement que $u^{\alpha}$ à l'infini, avec $\alpha<N /(N-2)$.

Introduction. In [1] we proved some results about the existence of at least one positive solution to the periodic parabolic problem

$$
\begin{aligned}
& \partial_{t} u-\Delta u=f(t, x, u) \quad \text { in } Q_{T}=(0, T) \times \Omega, \\
& u(T)=u(0) \quad \text { in } \Omega, \quad u=0 \quad \text { on } S_{T}=(0, T) \times \partial \Omega,
\end{aligned}
$$

where $\Omega$ is a bounded domain of $R^{N}, \partial \Omega$ is locally of class $C^{1}$, and $f$ is, roughly speaking, superlinear in the variable $u$.

To illustrate the results obtained in [1] we will consider the case $f(t, x, u)=$ $m(t, x) u^{\alpha}$ and we will denote by $\left(\mathrm{P}_{\alpha}\right)$ the corresponding problem. In [1] we proved that $\left(\mathrm{P}_{\alpha}\right)$ has at least one positive solution in $C^{2,1, \mu}\left(Q_{T}\right)(0<\mu<1)$ if $1<\alpha<$ $(N+1) /(N-1)$ and if $m$ satisfies the following conditions:

$$
\begin{gathered}
m(t, x) \geq m_{0}>0 \text { for all }(t, x) \text { in }(0, T) \times \Omega, \\
m(T, \cdot)=m(0, \cdot) \text { in } \Omega, \\
m \in C\left(Q_{T}\right), \quad m(\cdot, x) \in W^{1, \infty}(0, T) \text { uniformly in } x \in \Omega .
\end{gathered}
$$

Moreover if $m$ satisfies the technical assumption

$$
\left(\partial_{t} m(t, x)\right)^{-} \leq C_{\Omega, N} m(t, x) \text { for all }(t, x),
$$

where $C_{\Omega, N}$ is a positive constant which depends on $N$ and $\Omega$, then there exists a solution of $\left(\mathrm{P}_{\alpha}\right)$ for $1<\alpha<(3 N+8) /(3 N-4)$.

Received by the editors April 5, 1986 and, in revised form, September 8, 1986.

1980 Mathematics Subject Classification (1985 Revision). Primary 35K20, 35B10.

Key words and phrases. Parabolic nonlinear problems, superlinearity, periodic solutions. 
In this paper we improve these results in the case $1<\alpha<N /(N-2)$ as follows:

THEOREM 1. If $m$ satisfies conditions (1)-(3) and $1<\alpha<N /(N-2)$ if $N>2$, $\alpha<\infty$ if $N=2$, then there is at least one positive solution of $\left(\mathrm{P}_{\alpha}\right)$.

REMARK. Our conjecture is that problem $\left(\mathrm{P}_{\alpha}\right)$ possesses at least one positive solution for all $\alpha$ in $(1,(N+2) /(N-2))$, since in the case $m(t) \equiv m_{0}$ all the solutions of problem $\left(\mathrm{P}_{\alpha}\right)$ are necessarily stationary and there exists at least one for $\alpha$ in that interval.

This theorem will be proved by using a blow-up method which was introduced by B. Gidas and J. Spruck in [4] to obtain an $L^{\infty}$-estimate for positive solutions of semilinear elliptic equations. The same method has been lately used by Y. Giga and R. V. Kohn in [6] to prove some blow-up results for parabolic problems and by Y. Giga in [5] to study the asymptotic behavior of the global solutions of semilinear parabolic equations.

Notation. In all that follows we will denote by $C$ various positive constants, independent of the variables and functions considered in every case. Finally, either $\partial_{t}$ or $\partial / \partial t$ will denote the first derivative with respect to $t$.

The proof of Theorem 1 will consist of several lemmas and propositions. First we obtain $L^{\infty}\left(Q_{T}\right)$-estimates for the solutions of $\left(\mathrm{P}_{\alpha}\right)$; then we apply a standard topological degree argument to infer the existence of a positive solution for $\left(\mathrm{P}_{\alpha}\right)$.

REMARK. When $f$ has not the particular form considered above the assumptions we have to make on $f$ in order to insure the existence of a positive solution of (P) are the following:

$$
\begin{gathered}
f(\cdot, \cdot, s) \in C\left(Q_{T}\right) \quad \text { uniformly for } s \geq 0, \\
f(t, x, \cdot) \in W_{\text {loc }}^{1, \infty}\left(R^{+}\right), \\
f(T, \cdot, \cdot) \equiv f(0, \cdot, \cdot) \quad \text { in } \Omega \times \mathbf{R} \\
\liminf f(t, x, s) / s>\lambda_{1} \quad \text { as } s \rightarrow+\infty
\end{gathered}
$$

where $\lambda_{1}$ is the principal eigenvalue of $\partial / \partial t-\Delta$ in $Q_{T}$ with periodicity conditions in $t$ and Dirichlet boundary conditions (see [7]),

$$
\begin{gathered}
f(t, x, 0) \equiv 0 \quad \text { in } Q_{T} \\
\limsup f(t, x, s) / s<\lambda_{1} \quad \text { as } s \text { goes to } 0^{+}, \\
\text {either }\left|\partial_{t} f(t, x, s)\right| \leq C|f(t, x, s)| \text { for all } t, x, s, \text { or } \\
\left|(1 / s) \int_{0}^{s} \partial_{t} f(t, x, r) d r\right| \leq C f(t, x, s) s \quad \text { for all } t, x, s, \\
\lim f(t, x, s) s^{-\alpha}=h(t, x) \geq h_{0}>0 \text { as } s \text { goes to }+\infty, \text { for all } t, x .
\end{gathered}
$$

PROPOSITION 1. Assume that $m$ satisfies (1)-(3). Then there exists a positive constant $C$ such that if $u$ is a solution $\left(\mathrm{P}_{\alpha}\right)$ and $\alpha>1$, we have

$$
\int_{Q_{T}} f(t, x, u) d t d x \leq C .
$$

This proposition is proved in [1] and we will not redo it here. The main argument in this proof has already been used in [2] to treat general superlinear elliptic 
problems and lies in a method introduced in [4] to study the behavior of positive solutions of elliptic and parabolic problems near the boundary.

Proposition 2. Assume that $m$ satisfies (1)-(3) and that there exists a sequence of solutions of $\left(\mathrm{P}_{\alpha}\right),\left\{u_{k}\right\}$, in $C^{2,1}\left(Q_{T}\right)$ such that $\left\|u_{k}\right\|_{L^{\infty}}=M_{k} \rightarrow+\infty$. Then there exists a function $u$ in $C^{2,1}\left(R \times R^{N}\right)$ satisfying

$$
\begin{aligned}
& \partial_{t} u-\Delta u=u^{\alpha} \quad \text { in } R \times R^{N}, \\
& u \geq 0, \quad u \in L^{\infty}\left(R \times R^{N}\right), \quad u \neq 0 .
\end{aligned}
$$

PrOOF. This proof is already given in [1] and so we will only sketch it here. For all $k$ define $\lambda_{k}, \tau, y$, and $v_{k}$ as follows

$$
\lambda_{k}^{2 /(\alpha-1)} M_{k}=1, \quad y=\left(x-P_{k}\right) / \lambda_{k}, \quad \tau=\left(t-t_{k}\right) / \lambda_{k}^{2}, \quad v_{k}(\tau, y)=\lambda_{k}^{2 /(\alpha-1)} u_{k}(t, x),
$$

where $\left(t_{k}, P_{k}\right)$ is a maximum point of $u_{k}\left(u_{k}\left(t_{k}, P_{k}\right)=M_{k}\right)$.

1 st case. Assume that there is an accumulation point of $\left\{\left(t_{k}, P_{k}\right)\right\},(\mathbf{t}, \mathbf{P})$, in $[0, T] \times \Omega$.

The functions $v_{k}$ are well defined in the set $A_{k, \delta}=\left(-\delta / \lambda_{k}^{2}, \delta / \lambda_{k}^{2}\right) \times B\left(\delta / \lambda_{k}\right)$, $\delta$ small, where $B_{\beta}(M)=\left\{x \in R^{N} /|x-M|<\beta\right\}$ for any $M$ in $R^{N}, \beta$ in $R^{+}$.

Now since $\left\|v_{k}\right\|_{L^{\infty}}=v_{k}(0,0)=1$ for all $k$, the $v_{k}$ satisfy the equation

$$
\partial_{t} v_{k}-\Delta v_{k}=m\left(t_{k}+\lambda_{k}^{2} \tau, P_{k}+\lambda_{k} y\right)\left(v_{k}\right)^{\alpha} \quad \text { in } A_{k, \delta}
$$

and since $\lambda_{k} \rightarrow 0$ as $k$ goes to $+\infty$, we have the local estimates we need to pass to the limit in (7) and obtain the existence of $u$. Indeed we may get rid of $m(\mathbf{t}, \mathbf{P})$ by a simple scale change.

$2 n d$ case. All the accumulation points of the sequence $\left\{\left(t_{k}, P_{k}\right)\right\}$ lie on $[0, T] \times \partial \Omega$.

Let $(\mathbf{t}, \mathbf{P})$ be an accumulation point of the sequence of the points of maxima of $u_{k}$. We may assume without loss of generality that $\partial \Omega$ lies on the set $\left\{x_{N}=0\right\}$ near $(\mathbf{t}, \mathbf{P})$ and that the $N$ th coordinate of $P_{k}$ is positive. Then we define $v_{k}$ as above and we observe that this time the functions $v_{k}$ are well defined in the sets

$$
D_{\delta, k}=\left(-\delta / \lambda_{k}^{2}, \delta / \lambda_{k}^{2}\right) \times\left(B\left(\delta / \lambda_{k}\right) \cap\left\{y / y_{N}>-\left(d_{k} / \lambda_{k}\right)\right\}\right), \quad \delta \text { small, }
$$

where $d_{k}=d\left(P_{k}, \partial \Omega\right)$. Hence we may again obtain the local convergence of $\left\{v_{k}\right\}$ towards a function $v$ satisfying:

$$
\begin{aligned}
& \partial_{t} v-\Delta v=m(\mathbf{t}, \mathbf{P}) v^{\alpha} \quad \text { in } R \times\left\{y \in R^{N} / y_{N}>-s\right\} \\
& v=0 \quad \text { on } R \times\left\{y / y_{N}>-s\right\}, \quad v(0,0)=\|v\|_{L^{\infty}}=1, \quad v \geq 0
\end{aligned}
$$

where $s=\lim \left(d_{k} / \lambda_{k}\right)$ as $k$ goes to $+\infty$. If $\partial \Omega$ is not contained in $\left\{x_{N}=0\right\}$ near $(\mathbf{t}, \mathbf{P})$, one can always make a local coordinate change to have it. Doing so we see that $v_{k}$ is the solution of an elliptic equation more general than (15) in $D_{\delta, k}$. Then we pass to the limit as above and obtain that the limit function $v$ is a solution of an elliptic problem as (16), where $-\Delta$ has been replaced by a general elliptic operator with constant coefficients. By a conveniently chosen coordinate change we will be able to reduce this case to the preceding one, when (16) holds.

Now, if $s=+\infty$, we may conclude as in the first case. If on the contrary $s<+\infty$, then we may argue as follows: Let us denote $y^{\prime}=\left(y_{1}, \ldots y_{N-1}\right)$ : then

$$
\left(\partial v / \partial y_{N}\right)\left(\tau, y^{\prime},-s\right)=\lim \left(\partial v_{k} / \partial y_{N}\right)\left(\tau, y^{\prime},-\left(d_{k} / \lambda_{k}\right)\right) \quad \text { as } k \rightarrow+\infty
$$


and

$$
\left(\partial v_{k} / \partial y_{N}\right)\left(\tau, y^{\prime},-\left(d_{k} / \lambda_{k}\right)\right)=\lambda_{k}^{(\alpha+1) /(\alpha-1)}\left(\partial u_{k} / \partial x_{N}\right)\left(t, Q_{k}\right)
$$

for some $Q_{k} \in \partial \Omega$.

Then we have

$$
\left|\left(\partial v_{k} / \partial y_{N}\right)\left(\tau, y^{\prime},-\left(d_{k} / \lambda_{k}\right)\right)\right| \leq \lambda_{k}^{(\alpha+1) /(\alpha-1)}\|\nabla u\|_{L^{\infty}((0, T) \times \partial \Omega)} .
$$

But as we showed in [1], Proposition 1 allows us to prove that $\nabla u$ is uniformly bounded in a uniform neighborhood of $S_{T}$; hence $\partial v / \partial y_{N} \equiv 0$ on $\left\{y / y_{N}=-s\right\}$. Therefore we may define a new function $v_{0}$ in $C^{2,1}\left(R \times R^{N}\right)$ as follows:

$$
v_{0}\left(\tau, y^{\prime}, y_{N}\right)= \begin{cases}v\left(\tau, y^{\prime}, y_{N}\right), & y_{N} \geq-s \\ v\left(\tau, y^{\prime},-2 s-y_{N}\right), & y_{N}<-s\end{cases}
$$

and finally we obtain $u$ from $v_{0}$ by a scale change.

We prove next the result which allows us to improve the theorem given in [1].

Proposition 3. If $1<\alpha<N /(N-2)$ and $m$ satisfies (1)-(3) there exists a positive constant $C$ such that $\|u\|_{L^{\infty}((0, T) \times \Omega)} \leq C$ for all the solutions $u$ of $\left(\mathrm{P}_{\alpha}\right)$.

PROOF. If this result does not hold, then there exists a sequence of solutions of $\left(\mathrm{P}_{\alpha}\right),\left\{u_{k}\right\}$, satisfying $\left\|u_{k}\right\|_{L^{\infty}}=M_{k} \rightarrow+\infty$ as $k$ goes to $+\infty$. Then we apply Proposition 2 to obtain the existence of a function $u$ satisfying (14). Moreover, for every $k$ we have

$$
\begin{aligned}
\iint_{Q_{T}}\left(\partial u_{k} / \partial t\right)^{2} d t d x & =-\iint_{Q_{T}} m^{\prime}(t) u_{k}^{(\alpha+1)}(\alpha+1)^{-1} d t d x \\
& \leq C \iint_{Q_{T}} u_{k}^{(\alpha+1)} d t d x \leq C M_{k} \iint_{Q_{T}}\left(u_{k}\right)^{\alpha} d t d x
\end{aligned}
$$

and this last integral is bounded uniformly in $k$, as shown in Proposition 1.

On the other hand, for $k$ large and for every $R>0$ fixed we have

$$
\begin{aligned}
\iint_{[0, R] \times B_{R}}\left(\partial v_{k} / \partial \tau\right)^{2} d \tau d y & \leq 2 \lambda_{k}^{[4 /(\alpha-1)+2-N]} \iint_{Q_{T}}\left(\partial u_{k} / \partial t\right)^{2} d t d x \\
& \leq 2 C M_{k} \lambda_{k}^{[4 /(\alpha-1)+2-N]}
\end{aligned}
$$

Finally by the definition of $\lambda_{k}$ we see that $\left(M_{k} \lambda_{k}^{[4 /(\alpha-1)+2-N]}\right) \rightarrow 0$ as $k$ goes to $+\infty$ if $\alpha<N /(N-2)$. Hence $\partial v / \partial \tau \equiv 0$ in $R \times R^{N}$ and this implies that $v$ is independent of $\tau$ and satisfies the following elliptic problem:

$$
-\Delta v=v^{\alpha} \quad \text { in } R^{N}, \quad v \geq 0, \quad v \in L^{\infty}\left(R^{N}\right), \quad v \neq 0
$$

But this is impossible because, as it is shown in [4], problem (16) has no solution. Therefore such a sequence $\left\{u_{k}\right\}$ cannot exist and the proof is complete.

Now, before applying the degree argument which will allow us to finish the proof of Theorem 1, let us give the following auxiliary lemma.

LEMMA 4 . There exists a positive constant $\delta$ such that $\left\|u_{k}\right\|_{L^{\infty}((0, T) \times \Omega)} \geq \delta$ for all the solutions $u$ of $\left(\mathrm{P}_{\alpha}\right)$.

PROOF. If there were a sequence of solutions of the problem $\left(\mathrm{P}_{\alpha}\right),\left\{u_{k}\right\}$, satisfying $\left\|u_{k}\right\|_{L^{\infty}} \rightarrow 0$ as $k$ goes to $+\infty$, the function $w_{k} \equiv u_{k} /\left\|u_{k}\right\|_{L^{\infty}}$ would be a 
solution for the problem

$$
\begin{aligned}
& \partial_{t} w_{k}-\Delta w_{k}=m(t, x) u_{k}^{\alpha-1} w_{k} \text { in } Q_{T}, \\
& w_{k}(T)=w_{k}(0) \quad \text { in } \Omega, \quad w_{k}=0 \quad \text { on } S_{T},
\end{aligned}
$$

and for $k$ large enough $\left\|u_{1}^{(\alpha-1)}\right\|_{L^{\infty}}<\lambda_{1}(m)$, where $\lambda_{1}(m)$ is the principal eigenvalue of the problem

$$
\begin{array}{ll}
\partial_{t} u-\Delta u=\lambda m(t, x) u & \text { in } Q_{T}, \\
u(T)=u(0) \quad \text { in } \Omega, & u=0 \text { on } S_{T}
\end{array}
$$

(see [7]). Hence $w_{k}$ cannot exist, since its existence would contradict $\lambda_{1}$ 's definition. Therefore,

$$
\inf \left\{\|u\|_{L^{\infty}((0, T) \times \Omega)} \mid u \text { a solution of }\left(\mathrm{P}_{\alpha}\right)\right\}>0 \text {. }
$$

PROOF OF THEOREM 1. The topological argument we will use to conclude is exactly the same as in [1]. As we pointed out above the improvement obtained here is due to the fact that now we are able to obtain $L^{\infty}$-estimates under weaker assumptions on $m$.

Let us then sketch the end of the proof.

We define $K_{\lambda}: W^{1, q}\left(Q_{T}\right) \rightarrow W^{1, q}\left(Q_{T}\right)$ by $K_{\lambda} u=v$ if and only if:

$$
\begin{aligned}
& \partial_{t} v-\Delta v=\lambda m(t, x) u^{\alpha} \quad \text { in } Q_{T} \\
& v=0 \text { on } S_{T}, \quad v>0 \text { in } Q_{T}, \\
& v(T)=v(0) \quad \text { in } \Omega .
\end{aligned}
$$

We can easily verify that for $q$ large $K_{\lambda}$ is well defined and compact. Moreover by Lemma 4 there is no fixed point of $K_{\lambda}$ in the ball of radius $\rho$ and centered in the origin for some constant $\rho>0$ and for all $\lambda \in[0,1]$. Therefore $d\left(I-K_{1}, B_{\rho}, 0\right)=1$, where by $d(\cdot, \cdot, \cdot)$ we denote the Leray-Schauder degree.

On the other hand, we define another family of operators from $W^{1, q}\left(Q_{T}\right)$ into itself as follows: $T_{L} u=v$ if and only if $v$ is a solution of the problem

$$
\begin{aligned}
& \partial_{t} v-\Delta v=m(t, x)\left(v^{\alpha}+L v+L\right) \text { in } Q_{T}, \\
& v=0 \text { on } S_{T}, \quad v>0 \text { in } Q_{T}, \\
& v(T)=v(0) \text { in } \Omega .
\end{aligned}
$$

By Proposition 3, for every $L>0$ there exists $C(L)>0$ such that if $T_{L} u=u$, then $\|u\|_{L^{\infty}} \leq C(L)$. Furthermore it can be easily verified that for $L$ larger than a certain $L_{0}$, there is no fixed point of $T_{L}$. Then we can prove the existence of $M$ such that if $u$ is a fixed point of $T_{L}$ and $L \in\left[0, L_{0}\right]$, then $\|u\|_{L^{\infty}} \leq M$ and therefore it is obvious that $d\left(I-T_{L}, B_{M}, 0\right)=0$ for all $L$ in $\left[0, L_{0}\right]$. Finally since $K_{1}=T_{0}$ we see that $d\left(I-K_{1}, B_{M}-B_{\rho}, 0\right)=-1$, and the existence of a positive solution of $\left(\mathrm{P}_{\alpha}\right)$ follows immediately.

\section{BIBLIOGRAPHY}

1. M. J. Esteban, On periodic solutions of periodic parabolic problems, Trans. Amer. Math. Soc. 293 (1986), 171-189.

2. D. G. de Figueiredo, P. L. Lions and R. D. Nussbaum, A priori estimates and existence of positive solutions of nonlinear elliptic equations, J. Math. Pures Appl. 61 (1982), 41-63.

3. B. Gidas, W.-N. Ni and L. Nirenberg, Symmetry and related properties via the maximum principle, Comm. Math. Phys. 68 (1979), 209-243. 
4. B. Gidas and J. Spruck, A priori bounds for positive solutions of nonliner elliptic equations, Comm. Partial Differential Equations 6 (1981), 883-901.

5. Y. Giga, A remark on a priori bounds for global solutions of semilinear equations, Comm. Math. Phys. 36 (1987), 1-40.

6. Y. Giga and R. V. Kohn, Characterizing blow-up using similarity variables, Indiana Univ. Math. J. (to appear).

7. $\mathrm{P}$. Hess, On positive solutions of semilinear periodic-parabolic problems, Infinite-Dimensional Systems (Kappel-Schappacher, Ed.), Lecture Notes in Math., vol. 1076, 1984, pp. 101-114.

Laboratoire d'Analyse Numérique, Université P. et M. Curie, 75252 Paris Cedex 05, France 